hair dyeings. The results were not driven by personal hair dye use, or smoking (key subjects additionally evaluated for cotinine). Analysis of a randomly chosen hair waving product confirmed the presence of $\mathrm{o}$-and $\mathrm{m}$-toluidine.

Conclusions Our observations indicate that hairdressers are currently exposed to an established (o-toluidine), and a suspected (m-toluidine), human carcinogen from permanent hair dyes (including light colours) and unexpectedly also from hair waving.

\section{BACK SURGERY IN RELATION TO OCCUPATIONAL LIFTING. A COHORT STUDY BASED ON THE MUSCULOSKELETAL RESEARCH DATABASE AT THE DANISH RAMAZZINI CENTRE}

'Johan Hviid Andersen, ${ }^{2}$ Poul Frost, ${ }^{3}$ Jane Frolund Thomsen, ${ }^{2}$ Lone Donbaek Jensen, 'Susannne Wulff Svendsen. 'Danish Ramazzini Centre, University Deaprtment of Occupational Medicine, Herning, Denmark; ${ }^{2}$ Danish Ramazzini Centre, Department of Occupational Medicine, Aarhus, Denmark; ${ }^{3}$ Department of Occupational and Environmental Medicine, Bispebjerg Hospital, Copenhagen, Denmark

\subsection{6/oemed-2014-102362.102}

Objectives Controversies have long existed on causes for low back pain, and the role of occupational mechanical exposures, e. g. lifting has been debated for several decades. The aim of this study was to investigate if lifting is a risk factor for low back surgery.

Method The study is based on data from the Musculoskeletal Research Database at the Danish Ramazzini Centre, comprising nine previous studies on musculoskeletal symptoms in working populations, performed from 1993 to 2005 . The study was limited to participants aged 18-65, yielding 39258 individuals, 22 669 women (58\%) and 16589 men (42\%). Mean age at baseline was 42.9 years. Occupational mechanical exposures were assessed by a job exposure matrix linking job title to expert ratings of e.g. lifting, which was divided into three groups based on daily lifting: $0 \mathrm{~kg}$ (representing minimal exposure), 1-1000 kg, and $>1000 \mathrm{~kg}$. Cases of first time surgery for herniated lumbar disc $(\mathrm{n}=1025)$ or lumbar fusion $(\mathrm{n}=447)$ until 2012 were identified in the Danish National Patient Register. In preliminary analyses, risk estimates were obtained by logistic regression analysis, adjusting for age, gender, and study.

Results An exposure response relationship was seen for herniated lumbar disc: $\mathrm{OR}=1.2$ (95\% CI 1.0-1.4) for $1-1000 \mathrm{~kg} / \mathrm{day}$, and $\mathrm{OR}=2.2(1.9-2.6)$ for $>1000 \mathrm{~kg} /$ day. For lumbar fusion: $\mathrm{OR}=1.5(1.2-2.0)$ for $1-1000 \mathrm{~kg} / \mathrm{day}$, and $\mathrm{OR}=2.8(2.4-3.5)$ for $>1000 \mathrm{~kg} /$ day.

Conclusions Lifting was associated with later operations for both herniated lumbar disc and lumbar fusion. In further analyses, life style factors and occupational psychosocial exposures will be addressed.

\section{HOLES IN THE BURDEN ESTIMATES - SOME CAN BE FILLED, SOME MAYBE NOT}

${ }^{1}$ Tim Driscoll, ${ }^{2}$ Sally Hutchings, ${ }^{2}$ Lesley Rushton. 'Sydney School of Public Health, University of Sydney, Sydney, NSW, Australia; ${ }^{2}$ Imperial College London, London, UK

\subsection{6/oemed-2014-102362.103}

Objectives To consider the data shortcomings and methodological decisions involved in current burden of disease studies and the potential for these to be overcome and/or standardised.
Method Most burden of disease estimates require considerable assumptions or methodological decisions about factors concerning exposure, the appropriate relative risk to match with the exposure, and/or the size of the exposed population. These assumptions usually arise from a lack of data and could be largely overcome by the provision of better data. It is reasonable to expect that for some areas these data will improve with time, but for other areas the required data will probably never be available.

Other assumptions or methodological approaches vary depending primarily on theoretical considerations that are arguable and unlikely to ever be definitively solved by better data availability. Modelling may sometimes be of use but may not always be appropriate or practical and is still likely to involve some assumptions.

Results For example, some countries have reasonable estimates of asbestos exposure and some have good data on at least one asbestos-related outcome (mesothelioma incidence/mortality). How can this information be validly used for burden estimates where such data are poor?

Conclusions It is helpful to consider the extent to which burden estimates vary depending on the assumptions and methodologies involved when assessing the validity of estimates and their usefulness. Consideration of the potential for future improvements in data and better understanding of theoretical aspects should be an important input into the planning of future burden of disease work.

\section{MORTALITY AND MORBIDITY HEALTH IMPACT ASSESSMENT OF EXPECTED EXPOSURE TO PM10 DUE TO THE MAJOR CONSTRUCTION SITE FOR A LARGE INTERNATIONAL EXHIBITION}

${ }^{1}$ Michele Carugno, ${ }^{1}$ Giorgia Randi, ${ }^{2}$ Davide Campagnolo, ${ }^{2}$ Andrea Cattaneo, ${ }^{2}$ Domenico Maria Cavallo, 'Pier Alberto Bertazzi. ${ }^{1}$ Department of Clinical Sciences and Community Health, Università Degli Study Di Milano, Milano, Italy; ${ }^{2}$ Department of Science and High Technology, Università Degli Studi dell'Insubria, Como, Italy

\subsection{6/oemed-2014-102362.104}

Objectives To assess the short-term impact of expected exposure to PM10 due to a major construction site (Jan 2013-Jan 2015) on the health of the population residing in the seven towns nearby $(\mathrm{N}=235000)$.

Method Estimates of PM10 short-term effects on all-cause and cause-specific mortality and on selected causes of hospital admissions were estimated for a pre-construction period (2007-2011) using Poisson regression models. Expected PM10 concentrations at ground level were estimated applying the ISCST3 Gaussian dispersion model to forecast PM10 emission rates due to the site. Mean counts of the 2007-2011 deaths and hospitalizations were taken to estimate the expected numbers of health events. The 2013-2015 impact was evaluated in terms of numbers of attributable deaths and hospitalizations during the construction site progress, under several counterfactual scenarios.

Results Between 2013 and 2015, PM10 levels exceeding the mean PM10 pre-construction concentrations would be responsible for 0.54 attributable deaths $(0.13$ cardiovascular and 0.04 respiratory) and for 0.14 cardiac, 0.05 cerebrovascular and 0.51 hospital admissions. If considering the EU limit of $40 \mu \mathrm{g} / \mathrm{m}^{3}$, PM10 levels would be responsible for 11.06 attributable deaths (2.69 cardiovascular and 0.90 respiratory) and for 2.81 cardiac, 1.17 cerebrovascular and 10.89 respiratory hospital admissions. 